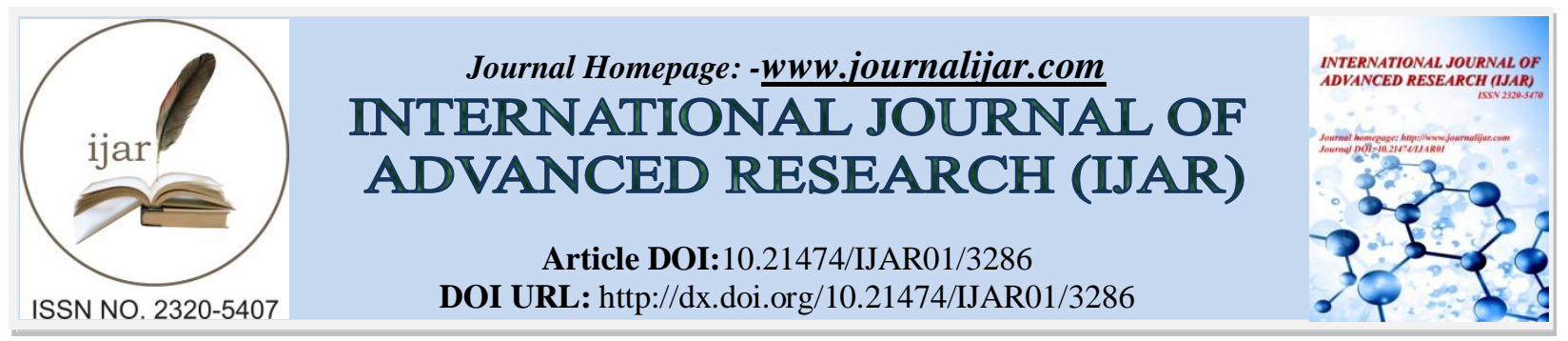

RESEARCH ARTICLE

\title{
PREVALENCE OF VITAMIN D DEFICIENCY IN TYPE 1 DM A CONTROL CROSS SECTIONAL STUDY HELD IN MIDDLE AND WEST REGIONS OF SAUDI ARABIA.
}

Olfat Z. Sambas ${ }^{1}$, Ahmad Z. Makeen ${ }^{2}$, Ammar S. Yamani' ${ }^{2}$, Alaa A. Alghamdi' and Rayan Z. Makeen ${ }^{2}$.

1. Pediatric Endocrinologist, Department of General Pediatric, Hera General Hospital, Makkah, Saudi Arabia.

2. Umm Al-Qura University, Makkah, Saudi Arabia.

\section{Manuscript Info}

Manuscript History

Received: 15 December 2016

Final Accepted: 18 January 2017

Published: February 2017

\begin{abstract}
This study compares theprevalence of Vitamin D deficiency inpediatricpatients of type 1 diabetes mellitus in thecentral and western regions of Saudi Arabia with children having no type 1 diabetes mellitus.

Research Methods: A control cross sectionalstudy conducted in AlYamamahHospital (CentralSaudi region) and Hera General Hospital (Western region). New cases or known casesof type1diabetes were recruited in the outpatient clinic and measurement of blood Vitamin Dlevel, PTH, Ca,Phosphorousand Alkaline phosphatase were done.

Result: The study showed that among type 1diabetic patients, $71.1 \%$ were severely Vitamin D deficient, $20.6 \%$ were moderately Vitamin D deficient, $8.1 \%$ were mildly Vitamin D deficientand $0.2 \%$ had normal Vitamin D levels. In the control group $42 \%$ were severely Vitamin D deficient, $18.8 \%$ were moderately Vitamin D deficient, $8.7 \%$ were mildly Vitamin D deficient and 30.4\% had normal Vitamin D levels.So overall nearly $100 \%$ of the children with Type 1 Diabetes Mellitus were deficient for Vitamin D as compared to $69.5 \%$ of the control children. Conclusion: - Vitamin D levels must be done for every child that hasproven Type 1 diabetes mellitus (T1DM). This will ensure early detection of Vitamin D deficiency and proper management. This will ultimatelyyield a better outcome and prognosis.
\end{abstract}

Copy Right, IJAR, 2017,. All rights reserved.

\section{Introduction:-}

Diabetes mellitus is a common disease and is especially prevalent (almost as an epidemic) in theMiddle Eastern countries. Many researchers have been highlighting the association of Type 1 diabetes and Vitamin D deficiency.Vitamin D isknown to regulate the functions of over 200 genes in our body and is essential for growth and development. (1).

Type 1 diabetes mellitus (T1DM) is the most commonly diagnosed type of DM in children and adolescents. It usually presents with symptomatic hyperglycemia and impliesthe immediate need for exogenous insulin replacement. (2).

Corresponding Author:-Olfat Z. Sambas.

Address:-Pediatric Endocrinologist, Department of General Pediatric, Hera General Hospital, Makkah, Saudi Arabia. 
Vitamin D3 is a fat soluble vitaminrequired for normal growth of all tissues originating from the ectoderm(e.g.bone, teeth,hair and skin). This is especially evident in children. Vitamin D deficiency can result in manifestationsof rickets in children and osteomalacia in adults.

The main source of Vitamin D is sun exposurethrough skin synthesis under the action of ultraviolet irradiation of ergosterol (D1) transferred to a crystalline compound called ergocalciferol (D2) and cholecalciferol (D3) which is formed in our body. Vitamin D is described as "the Sun Vitamin". It is a steroid with hormone like activity.Minor sources of Vitamin Dcome from dietary sources such as oily fish, meat and egg. Until 1980, the role of Vitamin D in the functioning of the immune system was unknown.

In recent years an effort has been made to understand possible non-calcemic roles of Vitamin $\mathrm{D}$, including its role in the immune system, particularly on $\mathrm{T}$ cell-medicated immunity. Vitamin D receptors are present in significant concentrations in the T lymphocyte and macrophage populations. However, there highest concentration were found in the immature immune cells of the thymus and the mature CD-8 T lymphocytes. The significant role of Vitamin D compounds as selective immune suppressants is illustrated by their ability to either prevent or markedly suppress animal models of autoimmune disease. Results show that 1.25 dihydroxyvitamin D3 can either prevent or markedly suppress experimental autoimmune encephalomyelitis $(3,4)$.

The incidence of diabetes is increasingly approachingan epidemic population in many countries including Saudi Arabia. There was a study done in 2010 in the Saudi province of Dhahran among a population of children younger than 15 years from the period of 1990-2007. Results showed an increase in the rate of diabetic patient from 18.05 per 100,000/year in the first 9 years to 36.99 per 100,000/year in the next 9 years. (5)

\section{Research Methodology:-}

From a control crosssectional study of all samples collected from Al Yamamah Hospital OPD as wellas Hera General Hospital OPD, we selected a sample of Saudi patient type 1 diabetes to measure theirtotal Vitamin D3 level in the blood. This laboratory investigation was done in two hospitals of different regions in SaudiArabia, the first one was inAlYamamah Hospital in Al-Riyadh which is the capital of Saudi Arabia andmarked by high temperatures during the day and low temperatures at night. Most of the country follows the pattern of the desert climatewithvery hot weather especiallyin Jun, July and Aug reaching temperatures up to 55 degrees Celsius. The second analysis was done in Hera General Hospital in Makkah which is the Islamic capital for Muslims that has a similar climateas Riyadh.

The patientsreferred to both centerseither as newly diagnosed type 1 diabetes based on clinical presentation for the disease,mostly DKA on admission, or rarely presented byhyperglycemia without DKA. Also known cases of T1DM being managed in the two hospitals were included in the study. Diagnosis of T1DM was confirmed by measuring Cpeptide.

The datawas collected in a period between Jan 2008 to Jun 2015. The sample consisted of 135patients aged between 1 to 16years. All patients were Saudi. Family history regarding T1DM or any risk factors for Vitamin D Deficiency (e.g. Sun exposure, nutritional history) were obtained from the parents. Vitamin D3 levels were measured by (Roche diagnostic Gmbh German) 411 Immunoassay analyzer(afterthe patients recovered from DKA episode). PTH, Ca, Phosphorousand Alkaline Phosphatase were measured as well.Patients then were followed monthly or every 3 months in OPD. Patients startedona conventional treatment in the form of NPH and Regular insulin. Patients presentedfromAl-Riyadh citydidn't like multiple daily injections $(50 \%)$ but remaining patients (50\%) started on multiple daily injections in the form oflong acting insulin (Lantus) and rapid actinginsulin (novorapid). The results of vitamin D3 levels were categorizedaccording tovitamin DCouncil (15).

Wedivided the levels into severe type, which isless than $30 \mathrm{nmol} / 1$, moderate typebetween $30-50 \mathrm{nmol} / \mathrm{l}$, and mild type between $50-70 \mathrm{nmol} / 1$ and normal more than $70 \mathrm{nmol} / 1$ whichis definedas an adequate vitamin levelwhere there are no symptoms or signs of vitamin D deficiency and the same was used in control group. We selected the control patient referred to OPD with complaint of non-thriving. The control group consisted of 83 patients.

There were certain Exclusion criteria:

$>$ Children Younger than 1 year whohave signsof rickets

$>$ Other endocrinal gland disease like (Hypo or Hyperaldosteronism)

$>$ Chronic disease (lungs, gastrointestinal disease) 


\section{Research sample:-}

There were a total of a 135 patients included in the study having type $1 \mathrm{DM}$. The mean age was 8.0356 years old (range from 1 to 16 years old) with BMImean 15.832 (11-25) P-value $<0.005$. There were $62(45.9 \%)$ males and 73 (54.07\%) females. The mean vitamin D3 level for all patients was $22.993 \mathrm{nmol} / \mathrm{l}(4-89 \mathrm{nmol} / \mathrm{l}) \mathrm{P}$-value $<0.005$. Mean HbA1c for all patients was 10.887 (1-17.5) P-value $<0.005$.

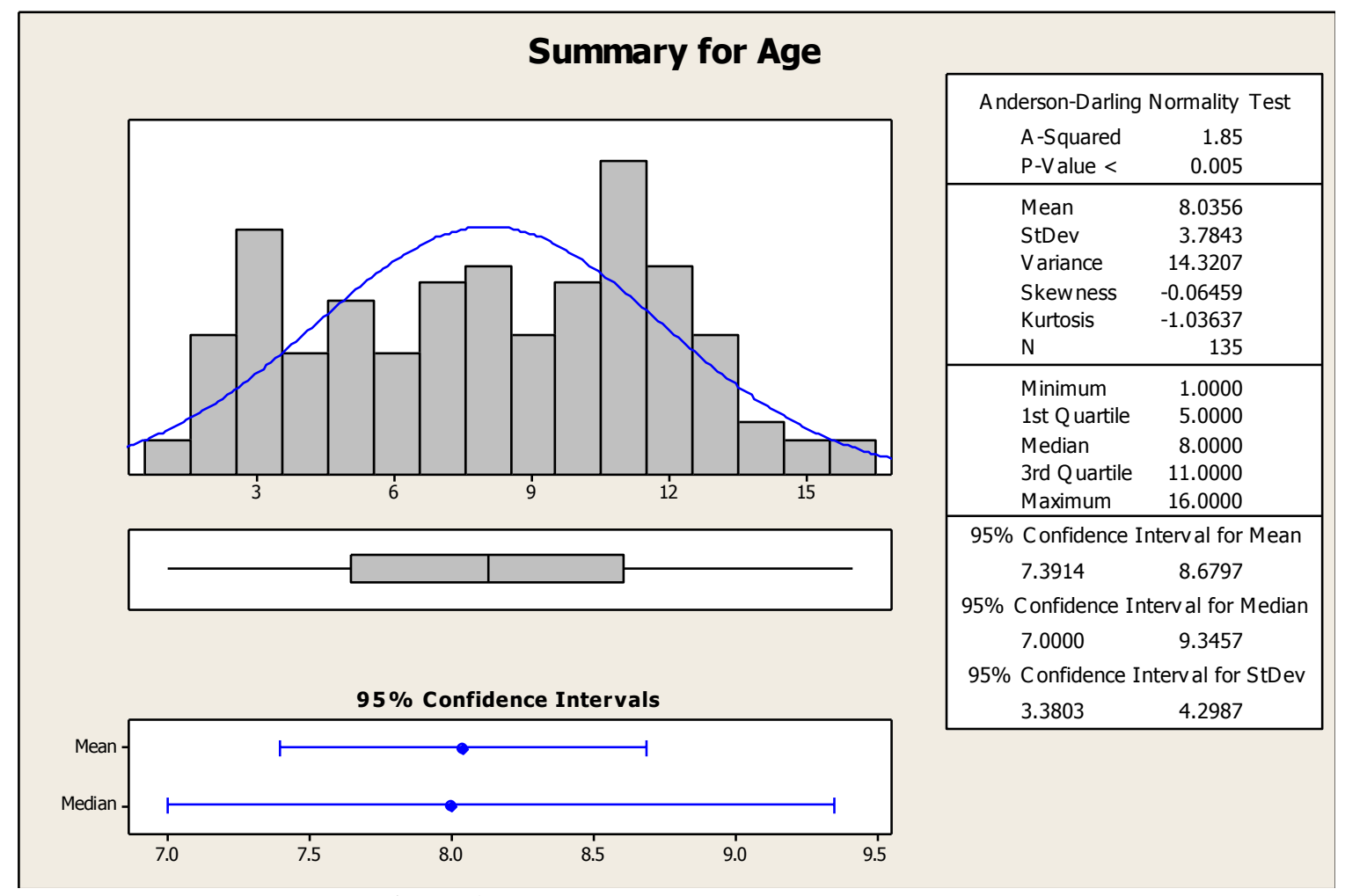

Figure 1:-Summary of Age of type one diabetes.

This diagrams shows the age vs. the number of type 1 diabetes patients. There are two clear peaks visible, one before the age of 5 and another between the ages of 9 and 15. Children are prone to infections around the age of the first peak ( 3 years) and around the second peak (11 years) they can suffer from pre pubertal hormonal secretion.

\section{Discussion:-}

There have been a lot of studies carried out showing the prevalence of vitamin D deficiency in type 1 diabetes compared with a control group. One of these studies is thenorth Indian study (6) which showed a Vitamin D deficiency in $91.1 \%$ of the subjects with diabetes, and in $58.5 \%$ of the healthy controls, $(60 \%)$ of the cases had severe Vitamin D deficiency compared to 8.3\% in the control group. Another study done in Qatar revealed that Vitamin D deficiency was considerably higher in T1DM children (90.6\%) compared to non-diabetic children (85.3\%). (7)

There was a significant difference found in the mean value of Vitamin D between T1DM and non-diabetic children in Egypt,among diabetic cases, 55\% were Vitamin D deficient; meanwhile 45\% of cases had normal Vitamin D levels $(P<0.01) .(8)$

In the T1DM children of Bin Abbas group, $64 \%$ were mildly Vitamin D deficient, $16 \%$ were moderately Vitamin D deficient, and $4 \%$ were severely Vitamin D deficient as compared with 52\% (mildly), 6\% (moderately), and $1 \%$ (severely) in the control group. Overall, $84 \%$ of the T1DM children, and 59\% of the healthy children were Vitamin D deficient (9). 
Alsoin another study of 129 Swiss children and adolescentswith type 1 diabetes, $78(60.5 \%)$ were Vitamin D deficient.In this study, Vitamin D deficiency was defined as a 25-hydroxy-Vitamin-D level below $50 \mathrm{nmol} / \mathrm{L}$. During the winter this number rose to 84.1\%. 25-hydroxy-Vitamin-D levels showed marked seasonal fluctuations. There was no correlation with diabetes control,despite the high prevalence of Vitamin D deficiency (10).

Prevalence of Vitamin D deficiency was $60.5 \%$ in a Swiss study(11), $43 \%$ in an Australian study (12), approximately $25 \%$ in an Italian study (13). In a cross-sectional study performed in Jeddah on 510 child ranged between 4-15 years, $13.7 \%$ had a normal Vitamin D level,58.8\% had relative deficiency,27.4\% had severedeficiency. Saudi and Yemeni patients weremore affected than Egyptian and other nationalities but all childrenwhich were included in the sample were not diabetic (14).

In our study, almost $100 \%$ of the children of type 1 diabetes had insufficient level of Vitamin D in comparison with (69.5\%)in control group. Severe deficiency was $71.1 \%$ in T1DM group and $42 \%$ in control group. Moderate deficiency $20.6 \%$ vs $18.8 \%$, mild deficiency $8.1 \%$ vs $8.7 \%$ and only $0.2 \%$ had sufficient Vitamin D levels vs $30.5 \%$.

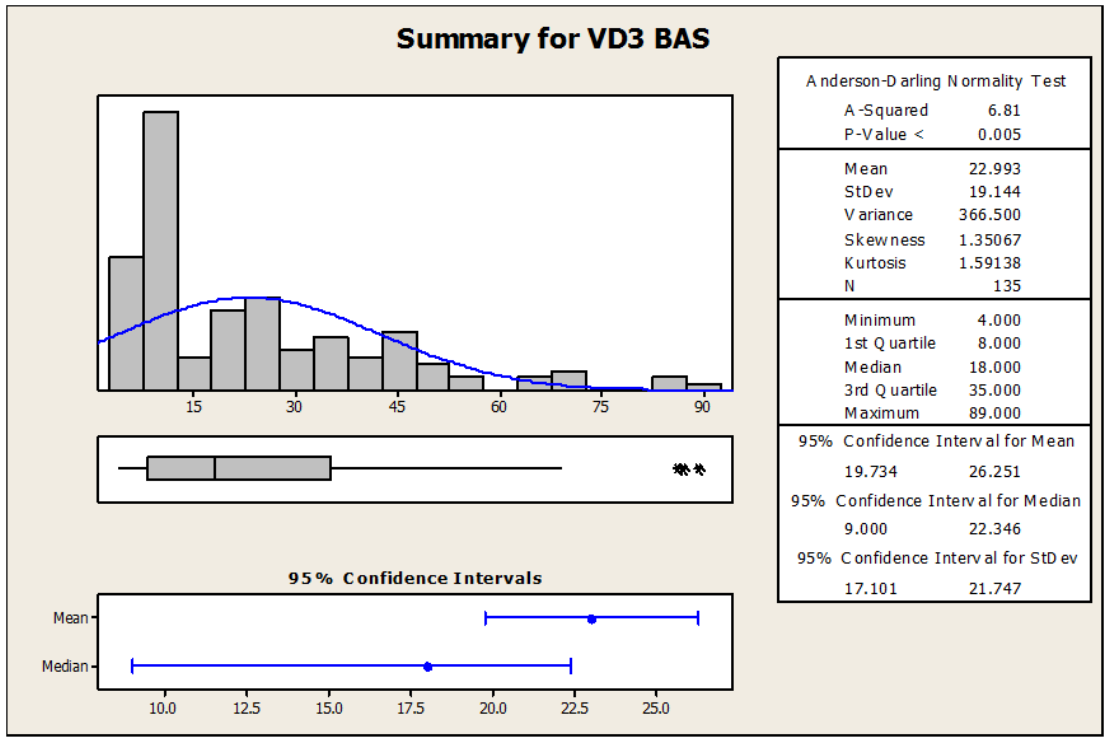

Figure 2:- Summary for VD3in type one diabetes.

This diagram shows the level of Vitamin D in nmol/l vs.the number ofT1DM patients.It is obvious that most of the patients lay in the insufficient area and they aremostly present with sever deficiency.

\section{Conclusion:-}

Vitamin D level should be a routine test for pediatric patients with Type 1 DM during diagnosis and follow up. Early detection of Vitamin D deficiency and appropriate management will lead to better prognosis and better quality of life, especiallythe effect on improving glycosylated hemoglobin(HA1c). This area is an evolving field of research and our knowledge regarding Vitamin D role in T1DM outcomes is not yet complete.

\section{References:-}

1. Lips P.Vitamin D physiology, Progress in Biophysics and Molecular Biology, 2006.

2. M. J. Haller, M. A. Atkinson, and D. Schatz, Type 1 diabetes mellitus: etiology, presentation, and management, Pediatric Clinics of North America, 2005.

3. Mohammad Abdul-Jabbar, Vitamin D deficiency in children living in Jeddah, Saudi Arabia, Saudi Medical Journal, 2010.

4. Brumbaugh, P. F., Haussler, M. R., Nuclear and cytoplasmic binding components for vitamin D, Life Science, 1975 . 
5. Kream, B. B., Reynolds, R. D., Knutson, J. C., Eisman, J. A., DeLuca, H. F., Intestinal cytosol binders of 1,25dihydroxyvitamin D3 and 25-hydroxyvitamin D3 Arch, Biochem. Biophys, 1976.

6. Riyaz Ahmad DagaI; Bashir Ahmad LawayI; Zaffar Amin ShahII; Shahnaz Ahmad MirI; Suman Kumar KotwalI; Abdul Hamid ZargarI. Arq Bras, High prevalence of vitamin D deficiency among newly diagnosed youth-onset diabetes mellitus in north India, EndocrinolMetab, 2012.

7. Bener A, Alsaied A, Al-Ali M, Al-Kubaisi A, Basha B, Abraham A, Guiter G, Mian M, High prevalence of vitamin D deficiency in type 1 diabetes mellitus and healthy children, ActaDiabetol, 2009.

8. Seham FA Azab, Safaa Hamdy Saleh, Wafaa F Elsaeed1, Sanaa M Abdelsalam1,Alshaymaa Ahmed Ali1 and Asmaa MH, Vitamin D status in diabetic Egyptian children and adolescents: a case-control study, Italian Journal of Pediatrics , 2013

9. Bin-Abbas B.S.; Jabari M.A.; Issa S.D.; Al-Fares A.H.; Al-Muhsen S, Vitamin D levels in Saudi children with type 1 diabetes, Saudi Medical Journal, 2011.

10. Janner M, Ballinari P, Mullis PE, Flück CE., High prevalence of vitamin D deficiency in children and adolescents with type 1 diabetes, Swiss Med Wkly, 2010.

11. Greer RM, Rogers MA, Bowling FG, Buntain HM, Harris M, Leong GM, et al, Australian children and adolescents with type 1 diabetes have low vitamin D levels, Medical Journal, 2007.

12. Pozzilli P, Manfrini S, Crinò A, Picardi A, Leomanni C, Cherubini V, et al, Low levels of 25-hydroxyvitamin D3 and 1,25-dihydroxyvitamin D3 in patients with newly diagnosed type 1 diabetes, HormMetab, 2005

13. Mansour MM, Alhadidi KM., Vitamin D deficiency in children living in Jeddah, Saudi Arabia, Indian J Endocrinal Metab, 2012.

14. Holick MF, Binkley NC, Bischoff-Ferrari HA, Gordon CM, Hanley DA, Heaney RP, Murad MH, Weaver CM; J, Endocrine Society. Evaluation, treatment, and prevention of vitamin D deficiency: An Endocrine Society clinical practice guideline, ClinEndocrinolMetab, 2011. 